\title{
A Fast Adaptation Mechanism for TCP Vertical Handover
}

\author{
Dagang Li, Kristof Sleurs, Emmanuel Van Lil and Antoine Van de Capelle \\ TELEMIC, Dept. of Electrical Engineering-ESAT, Katholieke Universiteit Leuven \\ Kasteelpark Arenberg 10, B-3001 Heverlee, Belgium \\ Email: dagang.li@esat.kuleuven.be
}

\begin{abstract}
In this paper we propose a vertical handover mechanism for TCP to deal with the delay and bandwidth change during the handover. The proposed mechanism relies on the interaction between the sender and the receiver during the handover and requires no a prior information of the new path. The adaptation is triggered by a handover notification at the receiver and finishes in about two round-trip time. Furthermore, it solves the problem of packet reordering and spurious retransmission timeout as well which are also common during vertical handovers. Simulation results show that our mechanism improves TCP performance in various vertical handover scenarios.
\end{abstract}

Index Terms - TCP, Handover, Congestion Control, Mobility.

\section{INTRODUCTION}

It is well-known that TCP does not perform well during a handover. A first reason is the possible packet loss during the outage time when the mobile node changes its network connection. Since TCP regards all packet loss as indication of congestion, it will back-off its sending rate unnecessarily. This problem can be readily solved in the network layer with lossless handover techniques such as buffer-and-forward and make-before-break. Unfortunately, this is not the end of problems. When the handover takes place between different type of networks, or in other words, when TCP experiences a vertical handover, the abrupt change in network delay and bandwidth will still affect its performance.

When the new path has a higher bandwidth or a lower delay, packets following the new path may overtake those in the old one and arrive at the receiver earlier. Consecutive out-oforder packets can generate enough duplicate ACKs to trigger false fast retransmission while no packet is really lost. On the other hand, when the new path has a lower bandwidth or a higher delay, packets following the new path may not be acknowledged in time, which causes premature RTOs at the TCP sender. All these wrongly triggered congestion responses not only cause unnecessary packet retransmission but also greatly decrease the TCP throughput regardless of the real network conditions. Furthermore, since TCP does not control the sending rate directly but only the number of in-flight packets using a sliding-window based mechanism, it will try to keep the same amount of packets in the network also after a handover. If the Bandwidth-Delay Product (BDP) is changed during a handover - which is very likely the case for a vertical one - TCP will either overrun or under-utilize the capacity of the new path. Because the Additive-Increase MultiplicativeDecrease (AIMD) scheme used in TCP for window adjusting is not fast enough to follow abrupt change of a handover, the performance of TCP will inevitably be degraded.

In this paper we propose a fast adaptation mechanism for TCP to address these vertical handover related problems. In our proposal we assume that the handover-induced packet loss is already taken care of in the network layer. Furthermore, a notification is assumed to be available from lower layer at the beginning of a handover, since as a transport layer protocol, TCP cannot detect the handover by itself. The trigger can be just a signal saying that the network interface has changed. We notice that indirect handover detection methods also exist, but they are not as fast and accurate as cross-layer triggers and are generally more complicated. Other than this trigger, no further information is required, such as active handover prediction or a prior knowledge of the new path, since they are difficult to acquire accurately in the current network environments.

The rest of the paper is organized as follows. Section 2 presents some related work on TCP vertical handovers. After that, our mechanism is presented and details are explained in section 3. Its performance is evaluated in section 4 with simulations, and section 5 concludes the paper.

\section{RELATED WORK}

The problem of TCP performance degradation during vertical handover has raised a lot of interest among network researchers [1]-[12]. Different aspects of the problem are studied and solutions are proposed accordingly.

Authors of [1]-[3] propose to hold the TCP sending during a handover and resume when the handover is finished. FreezeTCP [1] resumes with the same window size, which is not appropriate for vertical handover, as we discussed before. [2] solves this by letting TCP go through slow-start right after a handover. The idea is further developed in [3], where quickstart is used to negotiate the correct sending rate with traversing routers after the handover. For the problem of spurious retransmit timeout (RTO), [1], [2], [4] stop the retransmit timer or set it to a very large value during the handover, while [7], [8], [11] try to obtain the correct round-trip-time (RTT) estimation first and compute a new RTO based on it.

A lot more effort is put into adapting the TCP congestion window $c$ wnd to match the BDP of the new path after a vertical handover [4]-[7], [9], [10], [12]. Most of these techniques are based on a prior knowledge or estimation of the new path and feed the information back to the TCP sender to guide 
the adaptation, although the exact information and adaptation procedure may differ. The exception is [12], which proposes to equalize all the buffers in the network to absorb the change in BDP, but it is widely regarded as impractical.

Compared with the problems discussed above, packet reordering is much less studied. The reason might be that the affect is minor (cwnd only halved and limited packet retransmission) and TCP can recover from it by itself. Nevertheless, nodupack is proposed in [6] to enlarge the duplicate threshold dupThresh during the handover so more out-of-order packets are tolerated.

From the discussion we noticed two issues in the proposed techniques. First, no mechanism deals with all the major problems TCP may experience during a vertical handover. A better comprehensive solution should take care of all the main factors. Second, a lot of them assume a prior knowledge of the new path based on either previous measurement or the characteristics of the new access link. We argue that this knowledge can be inaccurate for the handed-over TCP traffic, because the new path traverses more than just the access network, and the effect of the dynamic cross traffic should be considered too. We believe an accurate and up-to-date knowledge of the new path can only be gathered by in-band end-to-end measurement during the handover.

\section{A FAST AdAPtATION MECHANISM}

In this section we will explain our adaptation mechanism for TCP vertical handover that handles packet reordering, spurious RTO and BDP change in a systematic way. It is true that not all of these problems will show up in each and every handover, but unlike many other techniques that explore different types of vertical handover (e.g. upward vs. downward handover) and treat them differently, our mechanism always perform the same handling procedure and does not need this categorization. The consistency simplifies the implementation and as we will see later, the overhead is neglectable.

\section{A. Design Considerations}

As mentioned in the introduction, we let the network layer handle the routing change to assure a lossless handover. To make the discussion easier, make-before-break is used as the example. Discussion over other schemes like buffer-andforward will be similar.

With both interfaces active, in-flight packets along the old path can still reach the receiver as normal. The proposed TCP adaptation is triggered when the receiver is notified of the handover, as shown in Fig. 1 at time $t_{0}$. The interaction between the sender and the receiver is also shown in this figure, where red lines are packets following the old path and blue lines are those following the new one. Specific measures are taken to deal with every potential problem we have identified so far. The details are discussed below.

1) Spurious RTO: We solve the problem of spurious RTO by sending two identical acknowledgements (ACKs) to the sender right after the handover is detected, one $\left(A C K_{0}\right)$ via the old path, the other $\left(A C K_{0}^{\prime}\right)$ via the new one. We call

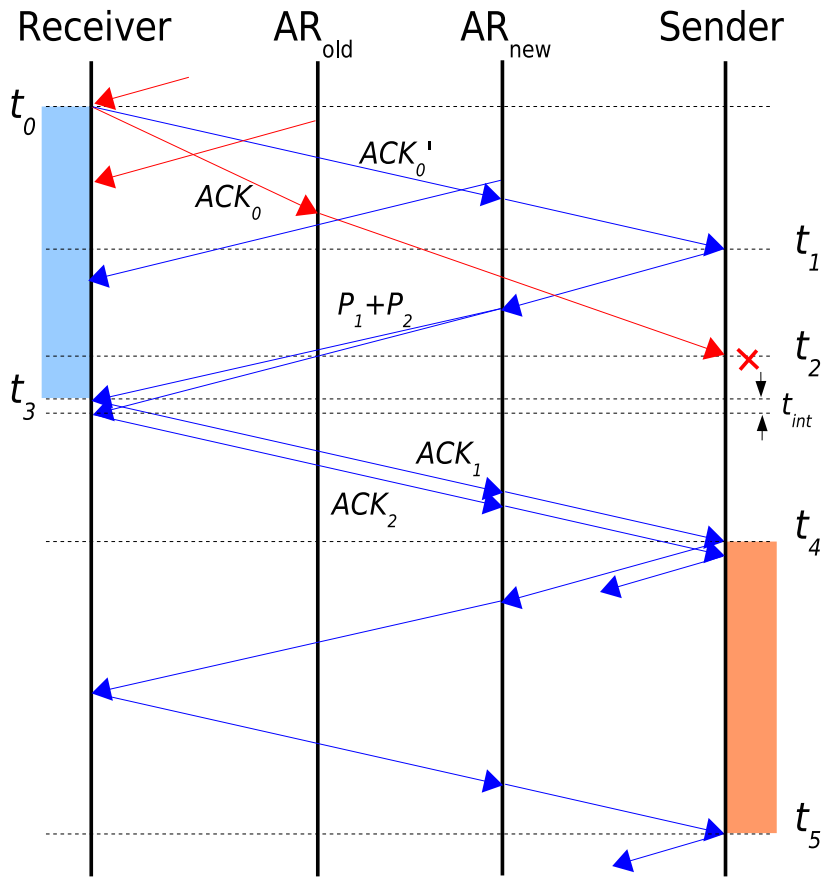

Fig. 1. TCP Fast Adaptation Mechanism

this method $d u p A C K$. This way $A C K_{0}$ will always arrive at the sender before RTO, and we use it to reset the RTO timer to the initial value ( $3 \mathrm{sec}$ in most systems) to prevent spurious RTO. On the other hand, because $A C K_{0}^{\prime}$ acknowledges a data packet from the old path but is returned via the new path, the RTT sample collected from it would be $R T T_{0}=\left(R T T_{\text {old }}+R T T_{\text {new }}\right) / 2$. Together with the smoothed RTT record $s R T T_{\text {old }}$ before the handover, the sender now has a good estimation of the RTT of the new path $R T T_{0}^{\prime}=$ $2 \cdot R T T_{0}-s R T T_{o l d}$, and can use it to reset the RTT record and calculate an appropriate RTO timer from it.

If $A C K_{0}$ reaches the sender later than $A C K_{0}^{\prime}$, then there is no danger of spurious RTO any more. In this case $A C K_{0}$ will be silently discarded, as shown in Fig. 1 .

2) BDP Probing: Vertical handover may be accompanied by BDP change and as discussed above, in-band end-to-end measurement is a better way to find out the right BDP along the new path. In our system it is called BDP probing and the procedure is as follows:

First, the sender sends two back-to-back packets $P_{1}$ and $P_{2}$ to the receiver after a handover. They will follow the new path to the receiver. If the access link of the sender is the bottleneck of the path, the ACKs of these packets will maintain the interval between them, otherwise the bottleneck link will separate them apart. Therefore, from the interval $t_{\text {int }}$ between $A C K_{1}$ and $A C K_{2}$ the sender gets an idea of the bottleneck bandwidth in the new path. Afterwards the TCP sender emits data packets periodically with $t_{\text {int }}$ in between (called packetpacing) and counts the number of in-flight packets in its congestion window $c w n d$, until the first ACK of such packets returns. This way at the end of BDP probing the TCP sender 
should have a rather accurate $c w n d$ that equals the BDP of the new path. With this measured cwnd, it then goes directly into congestion avoidance and resumes normal behavior. The packet pacing period is marked in light orange in Fig. 1.

If a more robust estimation of $t_{i n t}$ is desired, more than 2 back-to-back packets can be used for probing, but it also increases the possibility that the probing packets already overrun the buffer of the bottleneck in the new path. Another point to notice is that the estimated $R T T_{0}^{\prime}$ is not used with $t_{\text {int }}$ to calculate directly the desired window size, because the cwnd counted as in BDP probing is more reliable.

3) Packet Reordering: Packet reordering only happens when some of the first packets from the new path overtake packets in the old path and arrive earlier. In order to counteract packet reordering, it should first be identified and its degree calculated. Unfortunately, out-of-order packets can not be easily identified from lost packets. And although we can eliminate handover-induced packet loss in the network layer, congestion caused packet loss always exists (and actually is indispensable for TCP congestion control). Indeed, the solution from [6] only works when no packet loss is present.

In our mechanism we decide to avoid the effort of detecting the possible packet reordering, but just hold all the ACKs for a certain time from the beginning of the handover. During this period all arriving packets are silently received, including those out-of-order ones (if any). At the end of the period a congregate ACK is sent to the sender acknowledging the highest in-order sequence number, therefore all packet reordering before this ACK is tolerated. All following packets will be retransmitted, no matter whether they are already received, still on the way or dropped. This is a simple and reasonable solution as we will discuss soon, and this ACK holding mechanism also integrates nicely into the whole scheme. The ACK holding period is marked in light blue in Fig. 1.

4) TCP State and Residue Packets: TCP might be in one of its several states [13] when a handover happens, and its behavior upon the same arriving packet may differ depending on its current state. For example the cwnd will grow with different speed whether it is in slow-start or congestion avoidance. Since from BDP probing an appropriate cwnd is achieved, we do not want the old TCP state to be carried over to the new path and mess up with window adjusting, and that is why the TCP state is always set explicitly into congestion avoidance after BDP probing.

The same story goes with the packets that arrive from the old path after a handover. We call them residue packets. They bear implicitly information of the old path that should not affect the TCP congestion control after the handover. For example their arriving rate reflects the old bottleneck bandwidth, and a lost residue packet only indicates congestion in the old path. If they are acknowledged normally, the TCP sender will adapt to the wrong cause. Thankfully the ACK holding mechanism also helps in this case by hiding the false information from propagating to the sender. It ends when the first BDP probing packet arrives and only acknowledges the highest in-order data packets. All packets after this acknowledged sequence number

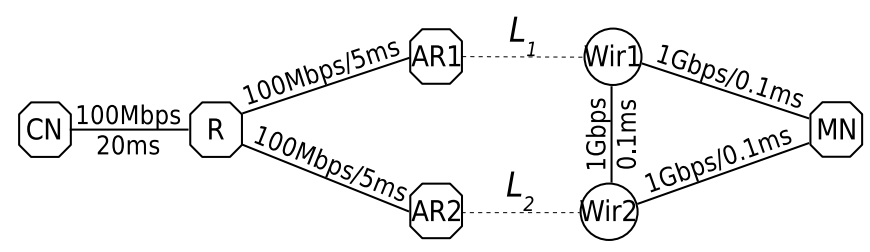

Fig. 2. Netork Topology used in Simulations

will be retransmitted starting from the packet pacing period, so all residue packets arriving afterwards can be just discarded. This way no embedded old information in the residue packets reaches the sender. This behavior is especially beneficial for the case when the old path has large delay and big buffer, or when TCP is in the state of self-clocking - it is better to retransmit the deep-buffered packets early than to wait until the buffer slowly depletes. In all other cases, the retransmitted packets will be very limited and will not noticeably affect the TCP throughput.

\section{B. Receiver Behavior}

Here we summarize the behavior of the receiver in our mechanism to show how the techniques discussed above are integrated together, with Fig. 1 as the reference.

Upon receiving the handover indication at $t_{0}$ the receiver sends two copies of the ACK of the first arriving data packet via the old and new path to inform the sender. After that it suppresses all ACKs and silently collects all incoming packets until $t_{3}$ when the first BDP probing packet arrives. It replies with the highest in-order sequence number and prepares the (re)transmission of all the following packets. From $t_{3}$ onwards, the receiver drops the old path and resumes the normal behavior of a TCP receiver.

\section{Sender Behavior}

The behavior of the sender is summarized as follows. As soon as the first $d u p A C K$ is received, the sender sets $c w n d$ to 2 and sends out two data packets back-to-back. If this ACK is from the old path, the sender also resets RTO timer to 3 sec. It then waits for the next ACK. Because the receiver suppresses all the ACKs until $t_{3}$, the next in-order ACKs should acknowledge the probing packets. When the first one arrives at $t_{4}$, the sender enters packet pacing and sends out packets periodically from the acknowledged sequence number with the measured interval from BDP probing. For each packet sent out, the $c w n d$ is increased by 1 , until the first such packet is acknowledged at $t_{5}$. The sender then enters congestion avoidance from then on with the cwnd at hand.

\section{Performance Evaluation}

The simple topology in Fig. 2 is used in our simulations. The bandwidth and delay of link $L_{1}$ and $L_{2}$ will be changed in different scenarios. A bulk Reno TCP flow is sent from the correspondant node $(\mathrm{CN})$ to the mobile node $(\mathrm{MN})$, while the latter moves from access router AR1 to AR2 at $20 \mathrm{sec}$ into the simulation by changing its interface from Wirl to Wir2 . 


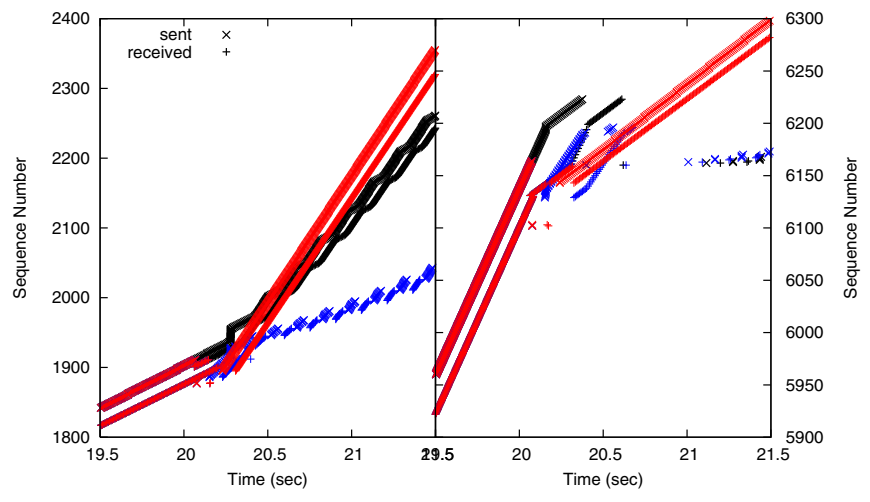

Fig. 3. Handover with changed bandwidth

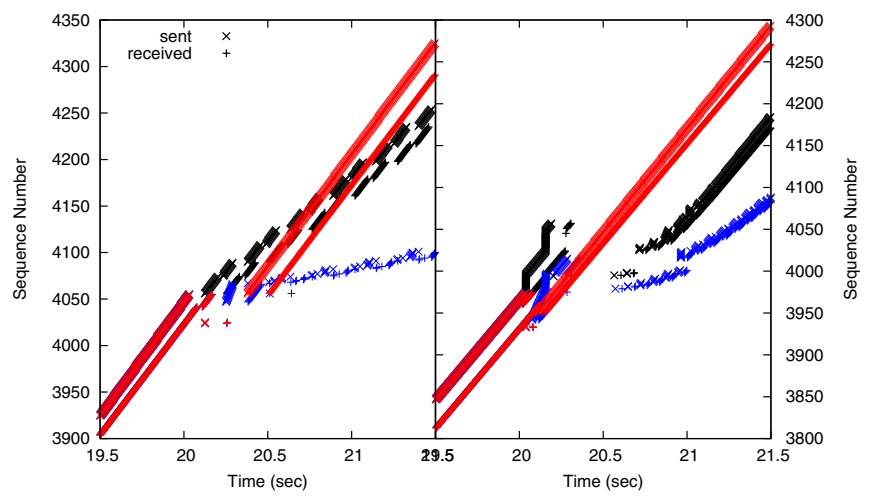

Fig. 4. Handover with changed delay

The buffer in each router is set according to the BDP of the link.

We only compare the proposed mechanism with pure Reno TCP and the Freeze-TCP variance from [2] (we call it vFTCP afterwards). The a prior knowledge based solutions are not compared since their performance greatly depends on the accuracy of the knowledge.

In Fig. 3 to Fig. 5, black marks are for Reno TCP, blue ones for vF-TCP and red ones for the proposed mechanism. Each half of the figure shows one direction of the handover, first from $L_{1}$ to $L_{2}$, then from $L_{2}$ to $L_{1}$.

Fig. 3 shows the case of a handover with changed bandwidth. In this scenario $L_{1}$ and $L_{2}$ have the same delay of $50 \mathrm{~ms}$ but different bandwidth of $1 \mathrm{Mbps}$ and $5 \mathrm{Mbps}$. It is clear that our mechanism can utilize the increased bandwidth faster and does not overshoot when the bandwidth decreases. Reno TCP overshoots severely and experiences heavy timeout when the bandwidth decreases. vF-TCP does not overshoot but adapts always slower than our mechanism.

In Fig. 4 we have a handover with changed delay. The bandwidth of $L_{1}$ and $L_{2}$ is the same at $2 \mathrm{Mbps}$, but the delay is $10 \mathrm{~ms}$ and $100 \mathrm{~ms}$ respectively. Here our mechanism also out-performs the other two.

In the last scenario $L_{1}$ is set to $500 \mathrm{kps} / 115 \mathrm{~ms}$ and $L_{2}$ to $2 \mathrm{Mbps} / 10 \mathrm{~ms}$, so the BDP over the whole path is unchanged after the handover. As shown in Fig. 5, Reno TCP seems

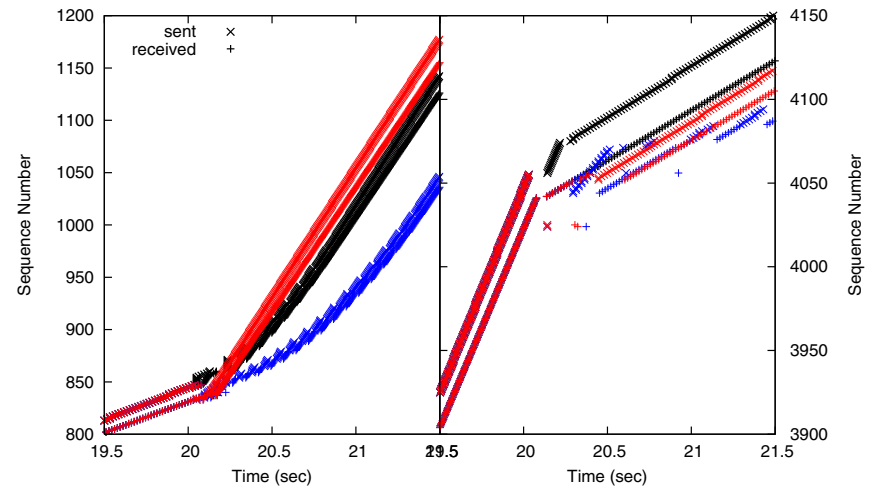

Fig. 5. Handover with unchanged BDP

to perform the best when handed-over from a $2 \mathrm{Mbps}$ link, because there happens to be enough BDP in the new path to absorb the short burst after the handover.

\section{CONClusion}

A fast adaptation mechanism is proposed to improve TCP performance during vertical handovers. The problems of spurious RTO, packet reordering and changed BDP are taken care of in the mechanism in a systematic way. Our mechanism does not depend on any a prior knowledge or specific type of the handover, therefore it improves the TCP performance in a large range of vertical handover scenarios.

\section{REFERENCES}

[1] T. Goff, J. Moronski, D. S. Phatak and V. Gupta, Freeze-TCP: A True Endto-end TCP Enhancement Mechanism for Mobile Environments, Proc. IEEE INFOCOM 2000, pp.1537-1545, March 2000

[2] S.-E. Kim and J. A. Copeland, TCP for Seamless Vertical Handoff in Hybrid Mobile Data Networks, Proc. IEEE GLOBEOCOM 2003, pp.661-665, Dec 2003

[3] P. Sarolahti, J. Korhonen, L. Daniel and M. Kojo, Using Quick-Start to Improve TCP Performance with Vertical Hand-offs, Proc. IEEE LCN'06, Nov 2006

[4] L. Daniel and M. Kojo, Using Cross-layer Information to Improve TCP Performance with Vertical Handoffs, Proc. IEEE AccessNets'07, Aug 2007

[5] X. Wu, M. C. Chan and A. L. Ananda, TCP HandOff: A Practical TCP Enhancement for Heterogeneous Mobile Environments, Proc. IEEE ICC 2007, June 2007

[6] W. Hansmann and M. Frank, On Things to Happen During a TCP Handover, Proc. IEEE LCN'03, Oct 2003

[7] K. Daoud and B. Sayadi, HAD: a Novel Function for TCP Seamless Mobility in Heterogeneous Access Networks, Proc. IEEE VTC-2007 Fall, Sept-Oct 2007

[8] H. Huang and J. Cai, Improving TCP Performance during Soft Vertical Handoff, Proc. IEEE AINA'05, March 2005

[9] Y. Matsushita, T. Matsuda and M. Yamamoto, TCP Congestion Control with ACK-Pacing for Vertical Handover, Proc. IEEE WCNC 2005, March 2005

[10] Y. Gou, D. AJ. Pearce and P. D. Mitchell, A Receiver-based Vertical Handover Mechanism for TCP Congestion Control, IEEE Trans. on Wireless Comm. Vol.5, No. 10, Oct 2006

[11] H. Rutagemwa, S. Pack, X. Shen and J. W. Mark, Robust Cross-Layer Design of Wireless-Profiled TCP Mobile Receiver for Vertical Handover, IEEE Transactions on Vehicular Technology, Vol.56, No.6, Nov. 2007

[12] A. Gurtov and J. Korhonen, Measurement and Analysis of TCP-Friendly Rate Control for Vertical Handovers, ACM MCCR 8(3), pp. 73-87, July 2004.

[13] A. B. Downey, TCP Self-Clocking and Bandwidth Sharing, Computer Networks, Vol.51, No.13, Elsevier, Sept. 2007 